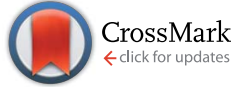

Cite this: RSC Adv., 2015, 5, 74353

\title{
Functionalization of nano-emulsions with an amino-silica shell at the oil-water interface $\uparrow$
}

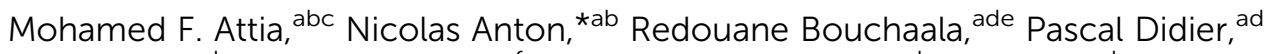 \\ Youri Arntz, ${ }^{\text {ad }}$ Nadia Messaddeq, ${ }^{f}$ Andrey S. Klymchenko, ${ }^{\text {ad }}$ Yves Mély ${ }^{\text {ad }}$ \\ and Thierry F. Vandamme ${ }^{\mathrm{ab}}$
}

Nano-emulsions are very promising nano-carriers with high potential for loading lipophilic drugs. However, the surface of oil nano-droplets is a dynamic oil/water interface stabilized by surfactants, and its chemical modification to graft ligands is highly challenging. In this study we developed a new protocol for modification of the nano-droplets surface through a silica shell terminated by amine functions. It enabled preparation of nanocapsules of 65,85 and $120 \mathrm{~nm}$ diameters with a surface coverage of ca. 2 amino groups per $\mathrm{nm}^{2}$. The nanocapsule surface was then functionalized ( $41 \%$ efficiency) by a model fluorescent ligand (coumarin blue) with a carboxylic function. The evidence for the successful grafting was provided by spectrofluorometry, Förster resonance energy transfer, atomic force microscopy coupled with fluorescence imaging and fluorescence correlation spectroscopy. This simple protocol for surface functionalization of the liquid/liquid interface of lipid droplets may constitute a real advance regarding potential applications that need efficient decoration of droplets with ligands.

Received 30th June 2015

Accepted 26th August 2015

DOI: $10.1039 / c 5 r a 12676 b$

www.rsc.org/advances half-life of the nanomedicines in the bloodstream. On the other hand, the selective accumulation in targets can also be improved by an active mechanism, involving ligand/receptor interactions. ${ }^{3}$ This approach also depends on the circulation time in blood of the nanoparticles (NPs), in order to ensure efficient ligand/receptor interactions. The higher the half-life, the better the chance of their interactions. Ligands grafted onto the NPs surface recognize the receptors and, as a result, NPs concentrate their active principles at the targeted site. Many ligands have already been proven to be effective for cancer active targeting and they can be grafted onto the surface at high concentrations. Typical examples are cyclic pentapeptides c(RGDfk) that, when grafted on the surface of polymeric PLGAPEG NPs (poly(D,L-lactic-co-glycolic acid)-block-polyethylene glycol), showed increasing in vivo accumulation in cancer cells. ${ }^{4}$ The co-encapsulation of anticancer active molecules results in specific treatment of the tumor. Other peptides like bombesin grafted onto gold NPs have shown an in vitro and in vivo cancer cell specificity allowing detection of tumors cells by X-ray imaging owing to the gold NPs accumulation. ${ }^{5}$ E-selectin peptides grafted on liposome surface, ${ }^{6}$ or 2-deoxy-D-glucose onto gold $\mathrm{NPs}^{7}$ have also shown interesting accumulation in cancer cells due to their enhanced glucose consumption. Specific targeting of pancreatic ductal adenocarcinoma can be performed through plectin-1 targeted peptides (PTP) at the surface of hybrid iron oxide/polymeric NPs. ${ }^{8}$ Another approach relies on the use of specific antibodies targeting cancer cells receptors, like anti-Her2 (ref. 9) or anti-CD4. ${ }^{10}$ Different other types of ligands could also be efficient for active targeting of 
tumors such as folic acid for which receptors are overexpressed in gastric cancer cells ${ }^{\mathbf{1 1}}$ or cationic polymers that target sialic acid overexpressed on colonic malignant tissues. ${ }^{\mathbf{1 2}}$

These examples clearly emphasized that specific targeting is efficient only if the surface concentration is high enough to provide a significant response or contrast in vivo. Active targeting is generally performed with polymeric or inorganic (particularly gold) NPs, because the surface chemistry performed on these systems is facile and efficient, providing a high number of available functional groups on the NP surface, and a high stability after ligand grafting. In the case of polymeric NPs, the functional groups (like carboxylic acids ${ }^{3 c, 4,13}$ ) are part of the polymer constituting the NP matrix. Therefore a large number of sites are available for ligands, which after grafting are strongly anchored onto the NP surface. Functionalization of gold NPs is generally performed through strong thiol/gold interactions in order to decorate the gold NP by thiolpolyethylene glycol-COOH molecules. ${ }^{10,14}$ In this case also, the reactive groups are strongly anchored to the NP core, presenting a high number of available reactive groups at the interface. Generally, the simple and efficient surface functionalization is one of the main advantages of polymeric ${ }^{\mathbf{1 5}}$ and inorganic NPS for active targeting strategies. In contrast, one of their main drawbacks is their limited capability in encapsulating active molecules, drugs or contrast agents, into the polymeric matrix or within the shell of inorganic NPs.

Other important family of nanomedicines is lipid nanodroplets, so-called nano-emulsions or nanocapsules. They are kinetically stable class of emulsions, in contrast to microemulsions that are thermodynamically stable. ${ }^{16}$ Nanoemulsions can be easily formulated and they enable high loading levels of guest molecules in their core. We recently obtained $^{\mathbf{1 7}}$ lipid nano-emulsions encapsulating in their lipid core, fluorescent probes with concentrations as high as $8 \mathrm{wt} \%$, opening new possibilities for single-particle tracking in vivo. ${ }^{\mathbf{1 7 b}}$ In addition, we formulated nano-emulsions as lipid reservoirs containing more than $50 \mathrm{wt} \%$ of iodine, and thus highly efficient for X-ray imaging. ${ }^{1,18}$ In addition to iodine, we cosolubilized in the same droplets significant amounts of a fluorescent dye, which allowed monitoring their cellular uptake. ${ }^{1}$ Nano-emulsions are also prospective for co-encapsulating drugs and contrast agents, and following their actual dosage in real time by imaging. In contrast, the active targeting of nanoemulsions is not presently well developed, due the technical difficulties related to the structure of the nano-emulsions. Indeed, oil droplets are stabilized by a monolayer of nonionic surfactants, so that there is no reactive groups for conjugation with ligands, and no possibilities for covalent anchoring of reactive chemical species as the liquid/liquid interface is a dynamic medium. Strategies such as post-insertion at the oil/ water interface of reactive function-bearing surfactants, like DSPE-PEG 2000-maleimide (1,2-distearoyl-sn-glycero-3-phosphoethanolamine- $N$-[maleimide (polyethylene glycol)-2000]) $)^{\mathbf{b}, \mathbf{1 9}}$ were developed, but the obtained surface modification is still largely below that of polymeric or inorganic NPs. The DSPE-PEG 2000-maleimide molecules are inserted in the surfactant monolayer, by incubating the lipid nano-droplets with a micellar solution of these molecules. However, in this approach the interfacial concentration is not controlled and is significantly lower compared to polymeric or inorganic NPs. In addition, the interfacial adsorption is definitively weaker than covalent bonds, and can even be reversible leading to ligand release into the biological medium.

In the present study, we propose a new and simple method to functionalize lipid nano-droplets. The idea is first to formulate nano-emulsions, and second to build amino-silica shell at the droplet interface. As a result, covalent grafting of ligands to the lipid nanocarriers becomes possible. The silica precursor, (3aminopropyl)triethoxysilane (APTES), beforehand solubilized in the oil core of the nano-emulsion droplets, reacted by ultrasounds with the aqueous phase at the droplets interface to generate the silica shell specifically at the interface. A significant part of the APTES' $\mathrm{NH}_{2}$ groups finally appears decorating the nanocapsules, oriented towards the aqueous bulk phase. We quantified the reactive $\mathrm{NH}_{2}$ groups by using the fluorescamine-based method, and further characterized the physicochemical properties of the functional nanocapsules by dynamic light scattering (DLS), transmission electron microscopy (TEM), and atomic force microscopy coupled with fluorescence microscopy (AFM-Fluo). Moreover, as a proof of concept, we grafted a fluorescent model ligand presenting activated $\mathrm{COOH}$ functions (coumarin blue dye) that react with the amino groups onto the capsules. Thus, we describe a robust approach to cover lipid droplets with a solid silica shell that enables their covalent functionalization.

\section{Experimental section}

\section{Materials}

The oil phase used in the preparation of nano-emulsions was exclusively Labrafac ${ }^{\circledR}$ WL 1349 (Gattefossé S.A., Saint-Priest, France), a mixture of capric and caprylic acid triglycerides as a model of parenteral-grade oil. Non-ionic surfactant (Kolliphor ELP®) from BASF (Ludwigshafen, Germany) was a kind gift from Laserson, Etampes, France. Kolliphor ELP (formerly named "Cremophor ELP") is a parenteral-grade non-ionic surfactant made by reacting ethylene oxide to castor seed oil at a molar ratio of $35 .{ }^{20}$ 3-Aminopropyltriethoxy silane (APTES), fluorescamine, ethanolamine, 7-(diethylamino) coumarin-3carboxylic acid $N$-succinimidyl ester (activated coumarin blue), Nile red (NIR), dry dioxane, dry dimethylformamide (DMF), and dimethylsulfoxide (DMSO) were purchased from Sigma. All chemicals used were of analytical grade and used without modification. Fetal bovine serum (FBS) was obtained from PAN Biotech (Aidenbach, Germany).

\section{Methods}

Formulation of amino-decorated lipid nano-emulsions. First, the silica monomer (APTES) is dissolved in the oil at different concentrations from $0.05 \mathrm{M}$ to $0.5 \mathrm{M}$. This \{oil + APTES $\}$ phase is then homogenized (vortex) 3 minutes at room temperature to obtain a transparent sample. It is then mixed with the non-ionic surfactant at different surfactant-to-oil 
weight ratio, SOR $=40 \%, 50 \%$ and $60 \%$, i.e. respectively $\left\{w_{\text {surf. }}\right.$ $\left.=0.32 \mathrm{~g} / w_{\text {oil. }}=0.48 \mathrm{~g}\right\},\left\{w_{\text {surf. }}=0.40 \mathrm{~g} / w_{\text {oil. }}=0.40 \mathrm{~g}\right\}$ and $\left\{w_{\text {surf. }}\right.$ $\left.=0.48 \mathrm{~g} / w_{\text {oil. }}=0.32 \mathrm{~g}\right\}$. This mixture is homogenized (vortex) during one minute and heated at $60{ }^{\circ} \mathrm{C}$ for only one minute to avoid premature reaction of APTES. The mixture is then homogenized for 10 seconds, and finally mixed with the water phase $(1.2 \mathrm{~mL}$, to have $60 \mathrm{wt} \%$ of water in the final nanoemulsion), and homogenized (vortex) for 5 minutes. The suspension of nano-emulsion droplets is placed in a sonication bath (Thermo Scientific, T310/H) at $35 \mathrm{kHz}$ during four minutes, and then homogenized (vortex) one minute, and this procedure is repeated 6 times. The result is a hydrolysis followed by a condensation of APTES, building the aminofunctionalized silica capsule at the oil/water interface.

Quantification of primary amines available on the nanocapsules. The fluorescamine-based method is a common procedure used for the quantification of primary amines in proteins or peptides. We adapted it for the nano-emulsion system. Before reaction with primary amines, the fluorescamine is not fluorescent, but after reaction with primary amines fluorescamine appear yellow and show fluorescence properties with absorption/emission around $288 \mathrm{~nm} / 490 \mathrm{~nm}$, respectively. Stock solution of fluorescamine $(10 \mu \mathrm{M}$ in anhydrous dioxane) was mixed with nano-emulsions and different APTES concentrations. The condensation reaction between fluorescamine and primary amines is very fast, being typically completed within seconds, so that the samples are mixed (vortex) for only one minute. Fluorescence intensities of the samples (diluted 30 times) were measured immediately after reaction by fluorescence spectroscopy. Each experiment was done in triplicate.

Grafting of activated coumarin blue (7-(diethylamino) coumarin-3-carboxylic acid $\mathrm{N}$-succinimidyl ester dye) on primary amines decorating the nanocapsules. In this step, our aim was to show that activated coumarin blue that reacted with the available $\mathrm{NH}_{2}$ groups at the surface of the nanocapsules as well as to quantify the grafting efficiency. To this end, we used the quantification data of available $\mathrm{NH}_{2}$ groups given by the fluorescamine method described above, and we added in the bulk phase the desired amount of activated coumarin blue able to react with $\mathrm{NH}_{2}$. Coumarin is a dye with absorption/emission at $394 / 473 \mathrm{~nm}$, respectively. A stoichiometric ratio (1:1) was tested, corresponding to $25 \mu \mathrm{L}$ of the stock solution of activated coumarin blue ( $32 \mathrm{mM}$ in dry dimethyl formamide) diluted in 1 $\mathrm{mL}$ of nano-emulsions having a concentration of available primary amines at $0.8 \mathrm{mM}$. The solution was then incubated for 24 hours under gentle stirring. Then, the sample was dialyzed through a $12 \mathrm{kDa}$ membrane (Spectra/Por®, Spectrum Europe B.V., Breda, the Netherlands) for another $48 \mathrm{~h}$, to ensure complete removal of excess unreacted dye (water changed three times). Finally, samples were characterized via fluorescence spectroscopy (see above), fluorescence correlation spectroscopy (FCS), single-particle measurement by TIRF microscopy, and atomic force microscopy coupled with fluorescence microscopy (AFM-Fluo).

Physicochemical characterization of nano-emulsions

Dynamic light scattering, zeta potential measurements. Size distributions and polydispersity indices (PDI) were measured by
DLS, along with zeta potentials, with a NanozS Malvern apparatus (Malvern, Orsay, France). The helium/neon laser, $4 \mathrm{~mW}$, was operated at $633 \mathrm{~nm}$, with the scatter angle fixed at $173^{\circ}$ and the temperature maintained at $25^{\circ} \mathrm{C}$. DLS data were analyzed using a cumulant-based method.

Transmission electron microscopy. The silica shell formed gives rise to a significant contrast in TEM. Therefore, samples were used without any staining agent and were diluted (1/100) with Milli-Q water. A drop of the suspension was placed on a carbon grid (carbon type-A, 300 mesh, copper, Ted Pella Inc. Redding, PA) and dried at $40{ }^{\circ} \mathrm{C}$. Observations were carried out using a Philips Morgagni 268D electron microscope.

Fluorescence spectroscopy. Absorption and fluorescence spectra were recorded on a Cary 4000 spectrophotometer (Varian) and a Fluorolog (Jobin Yvon, Horiba) spectrofluorometer, respectively. Fluorescence emission spectra were recorded at room temperature with 365,405 and $552 \mathrm{~nm}$ excitation wavelengths for fluorescamine-, coumarin blue-, and Nile red-loaded nanocapsules respectively. All fluorescence measurements were done using solutions with absorbance $\leq 0.1$. Förster resonance energy transfer (FRET) experiments were carried on nanocapsules decorated with coumarin blue as a donor. The acceptor dye Nile red was inserted into the oil core of the nanocapsules. FRET experiments were performed using the following preparation protocol: to $1 \mathrm{~mL}$ of MilliQ water we added $20 \mu \mathrm{L}$ of stock solution of Nile red (526 $\mu \mathrm{M}$ in DMSO), and $7.5 \mu \mathrm{L}$ of stock solution of silica-covered nano-emulsion decorated with coumarin (after dialysis, concentration was $600 \mu \mathrm{M}$ ). Control measurements were done with: (i) Nile red-loaded nanocapsules with silica shell but without coumarin grafting, and (ii) nanocapsules decorated with coumarin that did not contain Nile red.

Fluorescence correlation spectroscopy (FCS). FCS measurements were done on a two-photon platform including an Olympus IX70 inverted microscope. ${ }^{21}$ Two-photon excitation at $800 \mathrm{~nm}$ (15 mW laser output power) was provided by an Insight Deepsee femtosecond laser (Spectra-Physics). The measurements were performed in a 96 well plate, using a $200 \mu \mathrm{L}$ volume per well. The focal spot was set about $20 \mu \mathrm{m}$ above the bottom of the plate. Following the assumption that the NPs undergo a Gaussian diffusion in the two-photon excitation volume, the correlation function $G(\tau)$, calculated from the fluorescence fluctuations was fitted according to Thompson: ${ }^{22}$

$$
G(\tau)=\frac{1}{N}\left(1+\frac{\tau}{\tau_{\mathrm{d}}}\right)^{-1}\left(1+\frac{1}{s^{2}} \frac{\tau}{\tau_{\mathrm{d}}}\right)^{-\frac{1}{2}}
$$

where $N$ is the mean number of fluorescent species within the two-photon excitation volume, $\tau_{\mathrm{d}}$ is the diffusion time and $s$ is the ratio between the axial and lateral radii of the excitation volume. The NPs were diluted 200 -fold from the originally prepared nano-emulsion. Using 6-carboxytetramethylrhodamine (TMR from Sigma-Aldrich) in water as a reference $\left(D_{\mathrm{TMR}}\right.$ $\left.=421 \mu \mathrm{m}^{2} \mathrm{~s}^{-1}\right),{ }^{23}$ the diffusion coefficient, $D_{\text {exp }}$, of the nanoparticle was calculated by: $D_{\exp }=\tau_{\mathrm{d} \text { (capsules) }} / \tau_{\mathrm{d}(\mathrm{TMR})} \times D_{\mathrm{TMR}}$, where $\tau_{\mathrm{d}(\text { capsules) }}$ and $\tau_{\mathrm{d}(\mathrm{TMR})}$ are the measured correlation times for the nanocapsules and TMR, respectively. The hydrodynamic diameter $d$ of the nanocapsules was calculated with the Stokes- 
Einstein equation: $d=\left(2 k_{\mathrm{B}} T\right) /\left(6 \pi \eta D_{\text {exp }}\right)$, where $k_{\mathrm{B}}$ is the Boltzmann constant, $T$ is the absolute temperature $(293 \mathrm{~K})$ and $\eta$ is the viscosity of the bulk ( $1 \mathrm{mPa}$ ).

Atomic force microscopy coupled with fluorescence microscopy. The AFM/fluorescence microscope is a home-made association of an AFM composed of a SMENA Head driven by NTEGRA electronics (NT-MDT, Ru) combined with a wide-field fluorescence inverted microscope (Olympus IX-71). The fluorescence microscope is working in TIRF mode (Total Internal Reflection Fluorescence) with an oil immersion objective (NA $=1.49$, $100 \times$ ). A DPPS (cobolt) CW laser emitting at $488 \mathrm{~nm}$ was used to excite the NPs with a power of $3 \mathrm{~W} \mathrm{~cm}^{-2}$. Fluorescence imaging was performed using an EMCCD camera IXON 897 (ANDOR, UK). AFM images were acquired in the tapping mode, using a NSG03 (NT-MDT, RU) cantilever tip, with a spring constant of $0.9 \mathrm{~N} \mathrm{~m}^{-1}$, in liquid environment.

\section{Results and discussion}

Nano-emulsions present many advantages such as high encapsulation efficiency, ${ }^{17}$ stealth properties in the blood pool, ${ }^{1,18}$ and also facile formulation. ${ }^{\mathbf{1 6 , 2 4}}$ Therefore, the challenge in the functionalization of oil droplets relies in the postfabrication of a functional shell without affecting all these points. Several examples in the literature have shown the fabrication of a silica shell at the oil/water interface of emulsion droplets using TEOS (tetraethyl orthosilicate) as a silica precursor. $^{25}$ Here, we replaced TEOS for its derivative APTES bearing a primary amine as reactive group with the aim of building a silica shell decorated with $\mathrm{NH}_{2}$ functions. As schematically illustrated in Fig. 1, APTES is introduced in the oil phase during the spontaneous nano-emulsification process, to allow its spreading over all the population of forming droplets.

In general, the condensation of a silica precursor with TEOS uses a low $\mathrm{pH}$ in bulk as a catalyst. However, alternative methods using ultrasounds instead of chemical catalyst have been developed, ${ }^{25 a, 26}$ giving rise to the same TEOS interfacial condensation reaction. The advantage of the ultrasound approach is the possibility to use physiological $\mathrm{pH}$ and salt concentrations.

The obtained silica-treated nano-droplets, as imaged by TEM (Fig. 2(a)) appear as spherical structures with a diameter around 100-120 nm. In contrast, the control without silica (Fig. 2(b)) shows large and light oil puddles on the support, indicating that the droplets have merged during the drying process. Thus, while the sample drying on the carbon greed destroys the parent nano-droplets, the silica-treatment seems to strengthen the droplet structure. This corroborates the hypothesis that a silica framework or shell is built at the interface of the droplets. For the higher magnifications, we can note the malleability of the interface when the nanocapsules are stuck together, indicating that the nanocapsules are still deformable.

Next, we studied the size distribution, polydispersity, and zeta potential of the obtained nano-emulsions, as well as the impact of the silica concentration (APTES in oil) on these parameters. Fig. 3(a) shows a representative size distribution for the highest APTES concentration studied (0.5 M in oil). This distribution is centered at $115 \mathrm{~nm}$, in line with the size range observed on the TEM micrographs, and with a polydispersity index (PDI) of 0.16. In Fig. 3(b), we show the impact of the initial APTES concentration in oil on the nano-emulsion properties. Interestingly, the silica shell has no significant influence on the size distribution and PDI. This suggests that when solubilized in oil, APTES does not interfere with the spontaneous emulsification process.

The surface charge clearly decreases with increasing APTES concentrations, from an almost neutral value without APTES (around $-3 \mathrm{mV}$ ), up to around $-20 \mathrm{mV}$ above $0.25 \mathrm{M}$. This evolution of the surface properties likely reflects the density of silica at the water/oil interface, thus showing the gradual formation of the shell. These data also suggest that the shell covers the entire surface when $[\text { APTES }]_{\text {oil }} \geq 0.25 \mathrm{M}$. Considering the TEM pictures (Fig. 2) and the changes in the surface potential (Fig. 3(b)), likely related to the modification of the surface composition, we can conclude that the silica shell has effectively formed at the oil-water interface. Complementary studies on stability over time of the nanocapsules in FBS were carried out and reported in ESI Fig. S1. $\dagger$ The results did not reveal significant changes in the size over time, but showed a slight size increase for the higher dilution (1/100 and 1/1000), which could mean a weak aggregation. However, in the conditions of usual in vivo administration (e.g. 1/10 for X-ray imaging $^{\mathbf{1 , 1 8}}$ ) no significant changes were observed.

In this study, we used APTES instead of TEOS because it bears a primary amine, in order to decorate the nanocapsules formed after the silica poly-condensation. However it is obvious

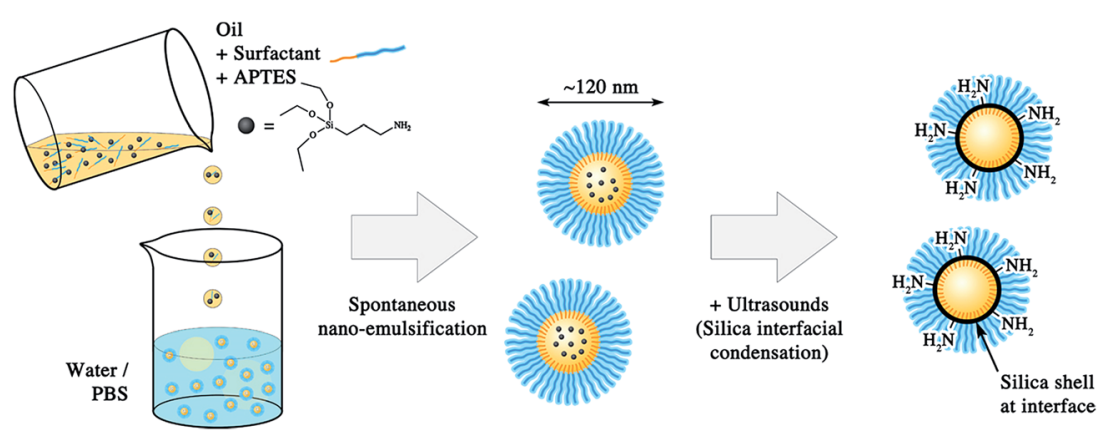

Fig. 1 General scheme describing the formulation of amino-functionalized nano-emulsion droplets. 

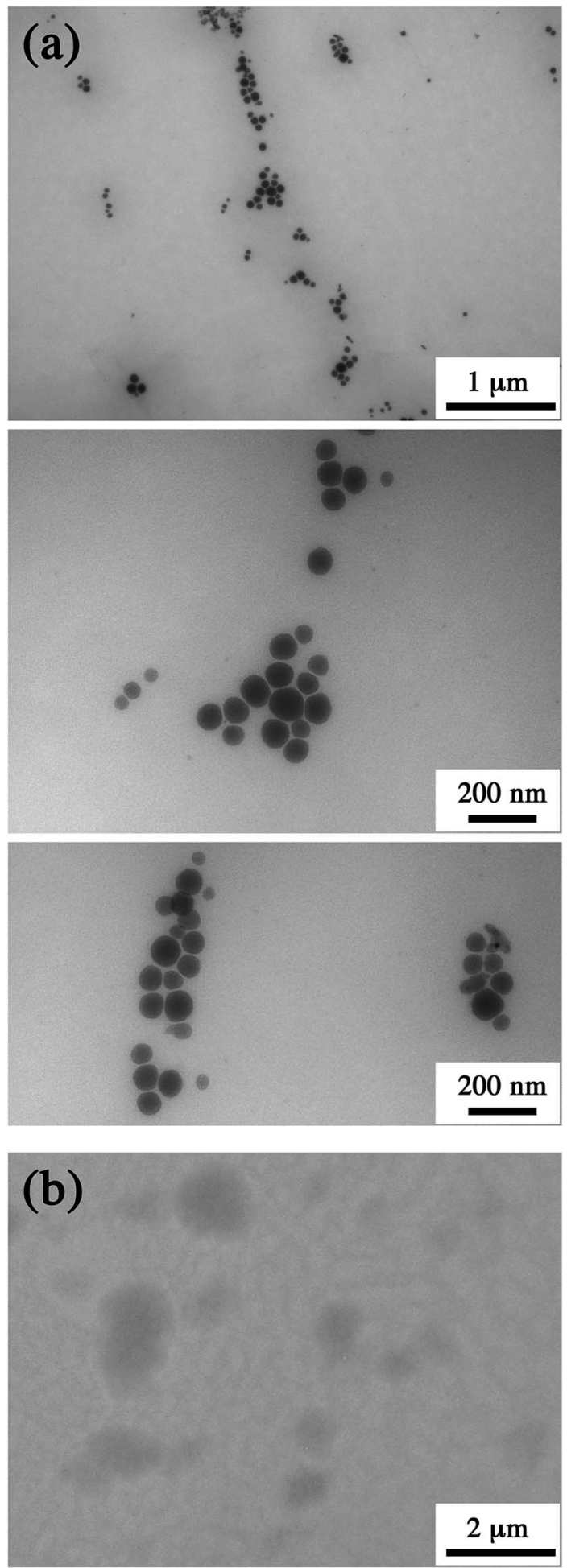

Fig. 2 (a) TEM pictures of nano-emulsion droplets after fabrication of the silica shell with $[\text { APTES }]_{\text {oil }}=0.5 \mathrm{M}$. (b) Control experiment, similar nano-emulsion formulation without silica shell.

that a significant part of the $\mathrm{NH}_{2}$ of the shell will be also entrapped into the oil core. To quantify the amino groups available for reaction at the nanocapsule interface, we used a method based on fluorescamine. This dye is routinely used in (a)
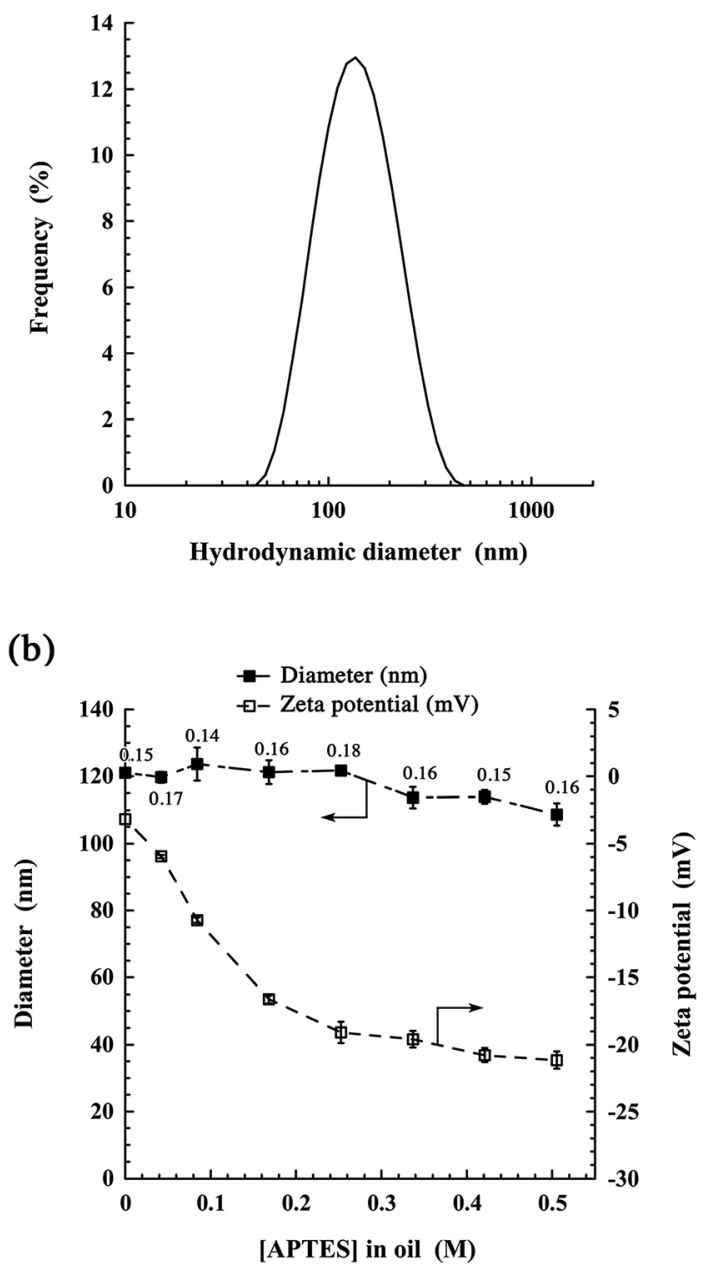

Fig. 3 Size distribution and zeta potential of silica- $\mathrm{NH}_{2}$ coated nanoemulsions. (a) Representative size distribution of the silica nanocapsules suspension, for [APTES] $]_{o i l}=0.42 \mathrm{M}$ obtained by DLS. (b) Effect of the APTES concentration on the size of the nanocapsules suspension (filled squares) and on their surface charge, measured through the zeta potential (open squares).

primary amine quantification of peptides or proteins, because it becomes fluorescent only after reaction with primary amines. To elaborate the protocol to the nanocapsules, we used a model water-soluble primary amine, ethanolamine. Different ethanolamine concentrations were mixed with increasing concentrations of fluorescamine, and the fluorescence intensity was measured (see scheme in Fig. 4, top). These experiments enabled us to calibrate the fluorescamine assay with our instrumental settings (ESI section†). It was found that for a fluorescamine concentration of $500 \mu \mathrm{M}$, the linearity region is obtained for ethanolamine concentrations in the range between 0 and $500 \mu \mathrm{M}$.

This calibration was used to determine the apparent $\mathrm{NH}_{2}$ concentration in the suspension of $\mathrm{NH}_{2}$-decorated nanocapsules. In order to be in the linear region, the nano-emulsions were diluted to a maximum theoretical $\mathrm{NH}_{2}$ concentration of $500 \mu \mathrm{M}$ and were mixed with $500 \mu \mathrm{M}$ fluorescamine. The 

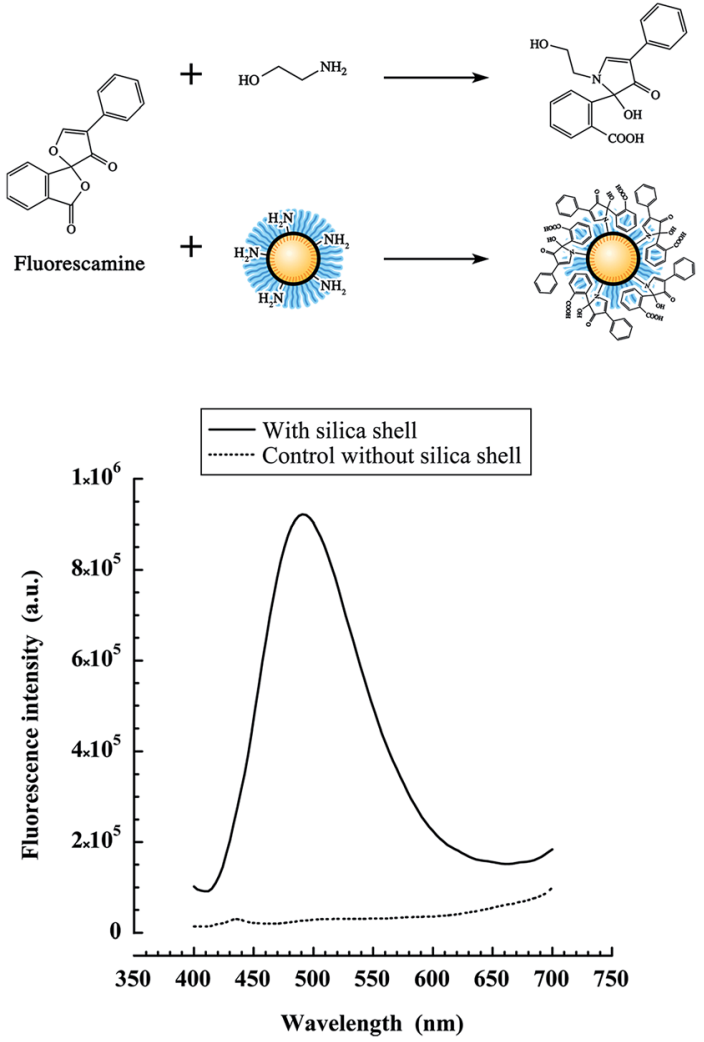

Fig. 4 (top) Schematic representation of the reaction of fluorescamine with ethanolamine and with primary amines onto the nanocapsules. (bottom) Emission spectra of fluorescamine (excited at 365 $\mathrm{nm}$ ) mixed (full line) with $\mathrm{NH}_{2}$-silica nano-emulsions and (dashed line) with blank nano-emulsions without silica shell.

fluorescamine reaction with the silica shell is schematically reported in Fig. 4 (top), giving rise to fluorescent nanocapsules. Nanoparticles with silica shell gave strong fluorescence, whereas no fluorescence was observed for blank nanoemulsions without silica shell (Fig. 4, bottom). This result confirmed the presence of reactive $\mathrm{NH}_{2}$ groups at the surface of the nanocapsules, as well as their accessibility for reacting with fluorescamine.

In addition, this method allowed evaluating the impact of the APTES (i.e. silica) concentration on the available $\mathrm{NH}_{2}$ groups at the interface, shown in Fig. 5 for 3 different nanocapsule diameters, 120, 85 and $65 \mathrm{~nm}$. The surface of the nanocapsules presents a very high coverage, up to $9 \times 10^{4}$ available $\mathrm{NH}_{2}$ groups per particle of $120 \mathrm{~nm}$ (i.e. 2 groups per $\mathrm{nm}^{2}$ ), prepared in the presence of $0.5 \mathrm{M}$ APTES in oil. The obtained value corresponds to around $50 \%$ of the amino groups introduced with APTES in the formulation (ESI section $\dagger$ ), indicating that half of the amine functions are entrapped in the droplets while the remaining half is available. As expected, the higher the silica concentration, the higher the number of amine functions per drop. Moreover, the larger is droplets size, the higher is the number of reactive groups. Finally, the straight lines fitting the experimental points indicate that the silica concentration at the interface is still growing and does not stabilize around

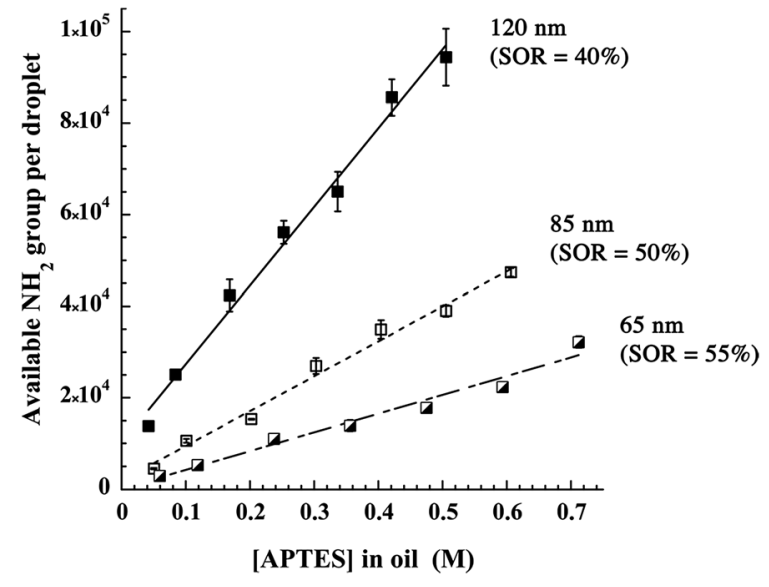

Fig. 5 Quantification, according to the fluorescamine method, of the available primary amine groups at the surface of the nanocapsules. Three different sizes of nano-emulsions were obtained by changing the SOR value as indicated. $n=3$.

[APTES $]_{\mathrm{oil}}=0.25 \mathrm{M}$ as it was shown with the zeta potential (in Fig. 3). This difference is probably because the zeta potential only reflects the surface coverage by the silica and once the surface is fully covered, the zeta potential does not change. On the other hand, increasing the silica concentration could increase the thickness and/or compactness of the layer thus increasing the total amount of amino groups.

In a next step, our objective was to graft model ligands on the available $\mathrm{NH}_{2}$ functions. As a proof of concept for the grafting of ligands bearing $\mathrm{COOH}$ function, we selected coumarin blue that presents a $N$-hydroxysuccinimide activated acid. After reaction for $24 \mathrm{~h}$, the samples are dialyzed for another $24 \mathrm{~h}$ to remove the free coumarin, and the emission of the samples is measured. The results reported in Fig. 6(a), show that coumarin ligands are grafted on the silica shell and not simply adsorbed on the nanoemulsion.

Surprisingly, the emission spectrum of coumarin blue is strongly red-shifted in comparison to the free dye, with a maximum emission wavelength at $554 \mathrm{~nm} v s .473 \mathrm{~nm}$ for the free dye in water. A possible explanation is that the fluorescence properties of the dye are modified by their high local concentration once they are grafted onto the capsule surface. The light absorption measurements at the maximum of the peaks, before and after dialysis allows us evaluating the proportions of dye grafted onto the nanocapsule, giving $41.5 \%$ grafted. That is to say around $3.7 \times 10^{4}$ coumarin dyes are grafted per particle. Such a huge local accumulation can modify the dye emission due to formation of ground or excited-state aggregates. To prove this hypothesis, we recorded the excitation spectra of the dyelabeled particles before dialysis (ESI $\dagger$ ). When recorded at 554 and $465 \mathrm{~nm}$, the excitation bands were both located around 400 $\mathrm{nm}$, with some blue shifted maximum for the former. These spectra were close to the corresponding absorption spectrum as well as to that of free coumarin dye in water. The small blue shift of the species emitting at $554 \mathrm{~nm}$ suggests a ground-state aggregation of the coumarin dyes at the particle surface. These 


\section{(a)}

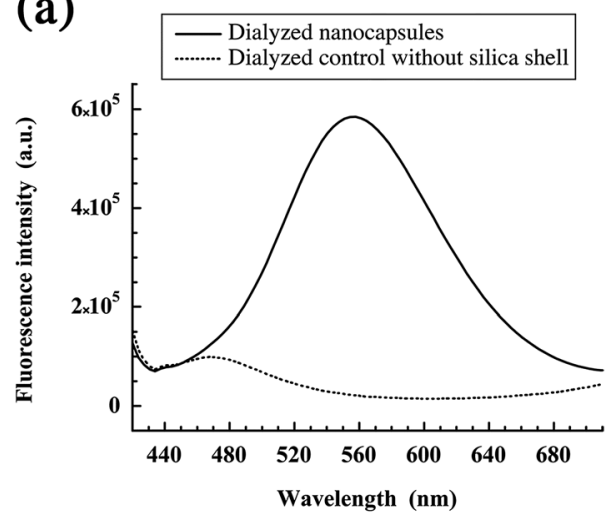

(b)
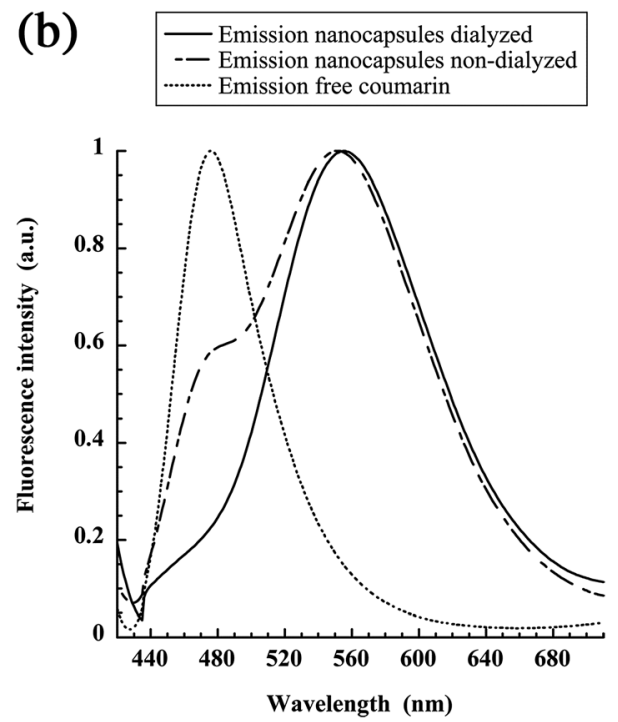

Fig. 6 (a) Emission spectra of coumarin blue (excited at $405 \mathrm{~nm}$ ) grafted on the surface of $\mathrm{NH}_{2}$-silica nano-emulsions with $[\text { APTES] }]_{\text {il }}=$ $0.5 \mathrm{M}$ after $24 \mathrm{~h}$ dialysis (full line) and of blank nano-emulsions without silica shell (dashed line). (b) Normalized emission spectra of coumarin blue grafted onto the surface of nanocapsules, with and without dialysis, and compared to free coumarin blue. Comparison of the spectra of the dye-labeled nanocapsules before and after dialysis shows the disappearance of the shoulder of free dye around $480 \mathrm{~nm}$.

aggregates may present a strongly red-shifted emission, as other dye aggregates. ${ }^{27}$ In addition, quantum yields ratio between free coumarin and grafted on nanocapsules (after dialysis) has been calculated from the spectra, giving the grafted dyes 5.3 times brighter than free dye in solution. This corroborates the drastic changes in the fluorescence properties after the surface conjugation.

Then, the dialyzed suspensions of nanocapsules decorated with coumarin, in which we have loaded the oil core with Nile red were studied with Förster Resonance Energy Transfer (FRET) experiments. Since the Nile red is homogeneously distributed in all the nanocapsules, our aim here was to study by FRET the co-localization of the two dyes in order to show that coumarin is grafted to all the nanocapsules. The control experiment with only coumarin gives a significant fluorescence peak. In the presence of Nile red as FRET acceptor, the

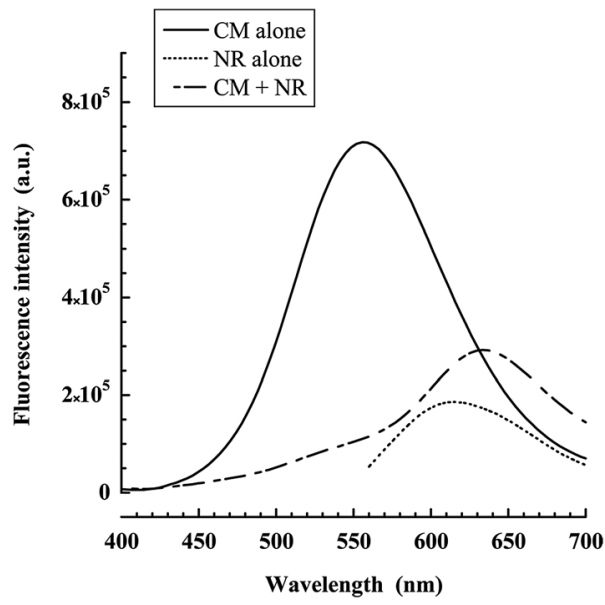

Fig. 7 Emission spectra for FRET experiments and controls. The studied systems are either nanocapsules only decorated with coumarin blue (CM alone), or nanocapsules only loaded with Nile red (NR alone), or finally nanocapsules decorated with coumarin blue and loaded with Nile red $(C M+N R)$; $[A P T E S]_{\text {oil }}=0.5 \mathrm{M}$.

fluorescence of coumarin is strongly inhibited giving rise to the fluorescence of the acceptor. Thus, the distance from coumarin to the closest Nile red molecules in the oil phase appears to be well below the Forster radius $(\sim 5 \mathrm{~nm})$.

Moreover, another physical method, combined AFM and fluorescence microscopy, shows the co-localization of the particles and the fluorescence signal of coumarin (Fig. 8). Indeed, the nanocapsules detected by AFM could be observed in the fluorescence images as bright spots, indicating that these nanostructures were labeled with the dye. However, the fluorescence spots appeared much larger due to diffraction limited resolution of fluorescence microscopy. The AFM micrographs

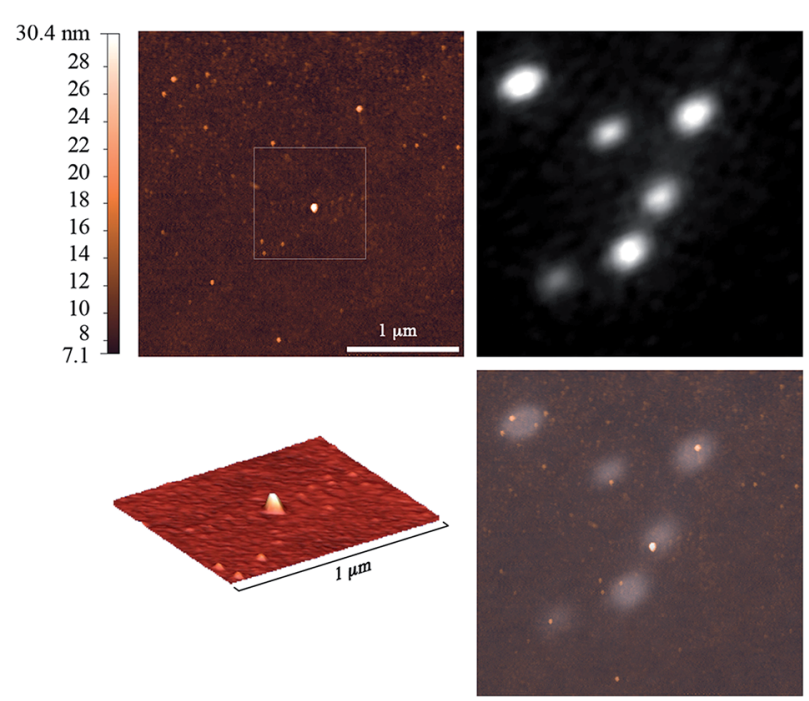

Fig. 8 Combined AFM and fluorescence microscopy of nanocapsules decorated with coumarin blue ([APTES $]_{\text {oil }}=0.5 \mathrm{M}$ ). Left: AFM micrograph with a 3D representation of the nanocapsules. Right: corresponding fluorescence acquisition showing the match between AFM and Fluorescence. 
Table 1 FCS data for coumarin blue-coated nanocapsules. Control refers to nano-emulsions without silica-shell and coated refers to nanocapsules with $\mathrm{NH}_{2}$-silica shell ([APTES] $\left.{ }_{\text {oil }}=0.5 \mathrm{M}\right]$ ). $\mathrm{N}$ is the average number of particles in the focal volume, $\tau_{\mathrm{c}}$ is the correlation time, and $\mathrm{Br}$ the brightness, is the ratio of photon count rate per capsule with respect to TMR. $\mathrm{Br} / \mathrm{Br}(\mathrm{TMR})$ is the brightness over the one of TMR

\begin{tabular}{lllll}
\hline & TMR $(5 \mathrm{~mW})$ & $\begin{array}{l}\text { NC }+ \\
\text { dye }(5 \mathrm{~mW})\end{array}$ & $\begin{array}{l}\text { TMR } \\
(10 \mathrm{~mW})\end{array}$ & $\begin{array}{c}\text { Free dye } \\
(10 \mathrm{~mW})\end{array}$ \\
\hline$N$ & 21.0 & 0.24 & 23.36 & 54.9 \\
$\tau_{\mathrm{c}}$ & 0.048 & 4.79 & 0.040 & 0.022 \\
$\mathrm{Br}$ & 2.27 & 38.72 & 7.54 & 0.20 \\
$\mathrm{Br} / \mathrm{Br}(\mathrm{TMR})$ & 1 & 17.1 & 1 & 0.027 \\
\hline
\end{tabular}

evidence the spherical shape of the particles, in line with the TEM image (Fig. 2). The fluorescent dots correspond well to the particles seen by AFM. Thus, AFM-Fluo further confirms that dialyzed samples show significant fluorescence originated from the nanocapsules.

To further confirm the successful surface modification of the nanocapsules, we studied by fluorescence correlation spectroscopy (FCS) the silica coated and non-coated nanocapsules after treatment with coumarin blue. FCS is a powerful technique to study fluorescent nanoparticles because it can provide simultaneously multiple parameters such as the correlation time, the number of particle per volume and the photon count rate, which provide access to the particle size, concentration and brightness, respectively. Corresponding values are reported in Table 1. Two powers of laser were adopted to compare nanocapsules with grafted dyes and free dyes, since on the one hand free dye is not sensitive enough at $5 \mathrm{~mW}$ (does not correlate) and on the other hand, we observed a strong bleaching at the grafted ones at $10 \mathrm{~mW}$. It can be seen that the grafted particles have a significantly higher correlation time than free species, TMR of free dyes. This indicates a bigger size calculated at $100.1 \mathrm{~nm}$, in agreement with DLS and TEM data. The same trend is observed with brightness, and to be comparable we consider the value of the brightness over the one of TMR as reference $(\mathrm{Br} / \mathrm{Br}(\mathrm{TMR}))$, giving the grafted ones around 633 times higher than free dye.

\section{Conclusion}

To summarize, the aim of this study was to propose a new method for functionalizing nano-emulsion droplets, after their formulation. Lipophilic silica precursor (APTES) was added to the oil before the formulation, and turned into silica shell after nano-droplet fabrication by sonochemistry that avoids addition of catalyst in the bulk phase. In comparison with existing methods to functionalize nano-droplets like post-insertion of functionalized lipids, this novel procedure brings real advantages: covalent grafting of functional groups at the nano-droplet surface i.e. strongly anchored on the silica nanocapsules, as well as a large number of reactive sites per capsule (up to $9 \times 10^{4}, \sim 2$ amino groups per $\mathrm{nm}^{2}$ ). The functional silica shell fabrication is simple, quick, and affects neither the formulation process nor the droplets size. Amino-group quantification allowed understanding the impact of formulation parameters, like the droplet size or APTES concentration, on the number of reactive $\mathrm{NH}_{2}$ groups decorating the resulting nanocapsules. Finally, the last part of the study was focused on the grafting of a model ligand though the reaction of an activated carboxylic acid (coumarin blue) on the amine functions available onto the nanocapsule surface. We achieved a grafting efficiency of around $41 \%$ and characterized the conjugate by various spectroscopic and microscopic methods, proving the concept of this new original protocol for functionalizing oil droplets. Nano-emulsions are a particularly interesting type of nano-carriers, able to solubilize a large range of active molecules or contrast agents, and their simple surface functionalization is a real step forward regarding potential applications like active targeting in vivo.

\section{References}

1 M. F. Attia, N. Anton, M. Chiper, R. Akasov, H. Anton, N. Messaddeq, S. Fournel, A. S. Klymchenko, Y. Mély and T. F. Vandamme, ACS Nano, 2014, 8, 10537.

2 (a) H. Maeda, J. Wu, T. Sawa, Y. Matsumura and K. Hori, J. Controlled Release, 2000, 65, 271; (b) S. Hirsjärvi, S. Dufort, G. Bastiat, P. Saulnier, C. Passirani, J. L. Coll and J. P. Benoît, Acta Biomater., 2013, 9, 6686.

3 (a) J. Tomasina, L. Poulain, E. Abeilard, F. Giffard, E. Brotin, L. Carduner, F. Carreiras, P. Gauduchon, S. Rault and A. Malzert-Fréon, Int. J. Pharm., 2013, 458, 197; (b) N. R. Jana, N. Erathodiyil, J. Jiang and J. Y. Ying, Langmuir, 2010, 26, 6503; (c) M. Soster, R. Juris, S. Bonacchi, D. Genovese, M. Montalti, E. Rampazzo, N. Zaccheroni, P. Garagnani, F. Bussolino, L. Prodi and S. Marchiò, Int. J. Nanomed., 2012, 7, 4797.

4 N. Graf, D. R. Bielenberg, N. Kolishetti, C. Muus, J. Banyard, O. C. Farokhzad and S. J. Lippard, ACS Nano, 2012, 6, 4530.

5 N. Chanda, V. Kattumuri, R. Shukla, A. Zambre, K. Katti, A. Upendran, R. R. Kulkarni, P. Kan, G. M. Fent, S. W. Casteel, C. J. Smith, E. Boote, J. D. Robertson, C. Cutler, J. R. Lever, K. V. Katti and R. Kannan, Proc. Natl. Acad. Sci. U. S. A., 2010, 107, 8760.

6 C. Wyss, S. C. Schaefer, L. Juillerat-Jeanneret, L. Lagopoulos, H. A. Lehr, C. D. Becker and X. Montet, Eur. J. Radiol., 2009, 19, 2487.

7 B. Aydogan, J. Li, T. Rajh, A. Chaudhary, S. J. Chmura, C. Pelizzari, C. Wietholt, M. Kurtoglu and P. Redmond, Mol. Imag. Biol., 2010, 12, 463.

8 K. A. Kelly, N. Bardeesy, R. Anbazhagan, S. Gurumurthy, J. Berger, H. Alencar, R. A. Depinho, U. Mahmood and R. Weissleder, PLoS Med., 2008, 15, e85.

9 J. F. Hainfeld, M. J. O'Connor, F. A. Dilmanian, D. N. Slatkin, D. J. Adams and H. M. Smilowitz, Br. J. Radiol., 2011, 84, 526. $10 \mathrm{~W}$. Eck, A. I. Nicholson, H. Zentgraf, W. Semmler and S. Bartling, Nano Lett., 2010, 10, 2318.

11 (a) X. Shi, S. Wang, S. Meshinchi, M. E. van Antwerp, X. Bi, I. Lee and J. R. J. Baker, Small, 2007, 3, 1245; (b) S. D. Weitman, R. H. Lark, L. R. Coney, D. W. Fort, 
V. Frasca, V. R. J. Zurawski and B. A. Kamen, Cancer Res., 1992, 52, 3396; (c) J. F. Ross, P. K. Chaudhuri and M. Ratnam, Cancer, 1994, 73, 2432.

12 A. K. Azab, J. Kleinstern, M. Srebnik and A. Rubinstein, Pharm. Res., 2008, 25, 379.

13 (a) E. Rampazzo, R. Voltan, L. Petrizza, N. Zaccheroni, L. Prodi, F. Casciano, G. Zauli and P. Secchiero, Nanoscale, 2013, 5, 7897; (b) M. Helle, E. Rampazzo, M. Monchanin, F. Marchal, F. Guillemin, S. Bonacchi, F. Salis, L. Prodi and L. Bezdetnaya, ACS Nano, 2013, 7, 8645.

14 J. Zhu, C. Waengler, R. B. Lennox and R. Schirrmacher, Langmuir, 2012, 28, 5508.

15 B. J. Kim, J. Bang, C. J. Hawker, J. J. Chiu, D. J. Pine, S. G. Jang, S. M. Yang and E. J. Kramer, Langmuir, 2007, 23, 12693.

16 N. Anton and T. F. Vandamme, Pharm. Res., 2011, 28, 978. 17 (a) A. Klymchenko, E. Roger, N. Anton, H. Anton, I. Shulov, J. Vermot, Y. Mely and T. F. Vandamme, RSC Adv., 2012, 2, 11876; (b) V. Kilin, H. Anton, N. Anton, E. Steed, J. Vermot, T. F. Vandamme, Y. Mely and A. S. Klymchenko, Biomaterials, 2014, 35, 4950.
18 X. Li, N. Anton, G. Zuber, M. Zhao, N. Messaddeq, F. Hallouard, H. Fessi and T. F. Vandamme, Biomaterials, 2013, 34, 481.

19 E. Bourseau-Guilmain, J. Béjaud, A. Griveau, N. Lautram, F. Hindré, M. Weyland, J. P. Benoit and E. Garcion, Int. J. Pharm., 2012, 423, 93.

20 J. Szebeni, F. M. Muggia and C. R. Alving, J. Natl. Cancer Inst., 1998, 90, 300.

21 J. P. Clamme, J. Azoulay and Y. Mély, Biophys. J., 2003, 84, 1960.

22 N. L. Thompson, Fluorescence correlation spectroscopy, in Topics in Fluorescence Spectroscopy Techniques, ed. J. R. Lakowicz, Plenum Press, New York, 1991, vol. 1, p. 337.

23 P. Didier, J. Godet and Y. Mély, J. Fluoresc., 2009, 19, 561.

24 N. Anton and T. F. Vandamme, Int. J. Pharm., 2009, 377, 142.

25 (a) S. H. Wu, Y. Hung and C. Y. Mou, Chem. Mater., 2013, 25, 352; (b) L. Spernath and S. Magdassi, Micro Nano Lett., 2009, $5,28$.

26 J. Ocotlan-Flores and J. M. Saniger, J. Sol-Gel Sci. Technol., 2006, 39, 235.

27 F. Würthner, T. E. Kaiser and C. R. Saha-Möller, Angew. Chem., Int. Ed., 2011, 50, 3376. 